“C 2019 IEEE. Personal use of this material is permitted. Permission from IEEE must be obtained for all other uses, in any current or future media, including reprinting/republishing this material for advertising or promotional purposes, creating new collective works, for resale or redistribution to servers or lists, or reuse of any copyrighted component of this work in other works." 


\title{
A Method of Generating Radiation Null for Periodic Leaky-Wave Antennas
}

\author{
Zheng Li, Member, IEEE, Shiqiao Zhang, Junhong Wang, Senior Member, IEEE, Yujian Li, Member, IEEE, \\ Meie Chen, Zhan Zhang, and Y. Jay. Guo, Fellow, IEEE
}

\begin{abstract}
A systematic method for generating radiation null region in the radiation pattern of periodic leaky-wave antennas is proposed using the theory of effective radiation sections. In this method, the aperture field is expanded into spatial harmonics using the mode expansion method, and the effective radiation sections of the harmonics are studied. Based on this, the radiation null region is introduced by suppressing the radiation of the effective radiation sections corresponding to the radiating mode. The proposed method is applied to a periodic-strip leaky-wave antenna. The validity of the proposed method is verified by both simulated and experimental results, showing an obvious radiation null in the prescribed angular range. This method has the advantages of easy calculation and implementation, and has little influence on the gain and beamwidth. It is illustrated that only simple modification to the antenna structure is needed to achieve nulling.
\end{abstract}

Index Terms-Effective radiation sections (ERSs), mode expansion, periodic leaky-wave antenna, radiation null.

\section{INTRODUCTION}

With the rapid development of wireless communication systems and radar systems, leaky-wave antennas (LWAs) have attracted increasing attention due to their inherent advantages of high gain, low profile, and low cost [1]. For certain applications, LWAs are required not to transmit or receive signal in certain directions in order to avoid unfavorable interference in these directions. To meet this requirement, a radiation null region is required in the radiation pattern.

There have been extensive investigations on the methods of generating radiation null region for uniform LWAs. For instance, for a LWA with long slot, tapered slot can be used to generate radiation null [2]. By tapering the slot continuously and accurately, the leakage constant and phase constant were simultaneously manipulated using a modified iterative Fourier technique, and then a wide and deep radiation null region can be achieved. In [3] a holographic design algorithm was proposed to control the complex aperture field of LWA, and was applied to the synthesis of radiation pattern with radiation null. In [4] a technique for generating prescribed radiation pattern was presented by adopting the OrchardElliott method, and was applied to a leaky-wave impedance surface to achieve radiation null. In [5] and [6] a transmission line model and global optimization method were proposed for high accuracy in pattern synthesis for LWA to realized null. Similar approach can also be used to achieve lower side lobe levels (SLL) [3]-[8]. Note that all the mentioned methods were applied to the LWAs with continuous line-source radiation apertures. Also, some of the methods can be utilized to the modulation of periodic LWAs for pattern synthesis [9]-[11]. On the other hand, LWA can be treated as an antenna array of approximated elementary radiators with a short sampling distance [12]-[14], so the existing array techniques [15]-[17] might be useful to synthesize radiation patterns of LWAs. As originally explained in [2], [11]-[14], the condition is that the sampled elementary radiators

This work was supported by NSFC Project under grant no. 61771038 and 61871025, and the Australia Research Council under Grant DP160102219.

Z. Li, S. Q. Zhang, J. H. Wang, Y. J. Li, M. E. Chen, and Z. Zhang are with the Key Laboratory of All Optical Network and Advanced Telecommunication Network of Ministry of Education and the Institute of Lightwave Technology, Beijing Jiaotong University, Beijing 100044, China (e-mail: lizheng@bjtu.edu.cn).

Y. J. Guo is with the Global Big Data Technologies Centre, University of Technology Sydney, Ultimo, NSW 2007, Australia. that form the discretized LWA aperture are not independent, but they have coupling effect from the leaky mode. The LWA can be tapered or modulated, but in a slight or smooth manner to avoid unwanted radiation due to strong discontinuities (reflected and diffracted waves). Thus one must satisfy these conditions of a smooth tapered leaky wave in order to directly relate the radiation pattern of a modulated LWA with its geometry from the leaky-mode perspective. The existing pattern synthesis methods for antenna arrays (which also have periodic structures) cannot be adopted directly for periodic LWAs, such as the iterative FFT [15], convex optimization [16] and stochastic optimization algorithms [17, 18]. The main reason is that, since the operation of periodic LWAs is based on travelling waves, it is difficult to control the phase and magnitude independently and arbitrarily in one periodic cell in the way one deals with antenna arrays [15]-[18]. Therefore, there is a need to find effective ways to realize nulling for periodic LWAs.

In this paper, a method based on the effective radiation sections (ERSs) [19] is developed for generating radiation null for periodic LWAs. The concept of ERSs provides a new insight into the radiation mechanism of uniform traveling-wave structures. It demonstrates that, for any given direction, the far field of a travelingwave structure can be calculated by the ERSs while other sections have little contribution. In [20] a low SLL and a radiation null region were achieved for a uniform slot LWA by adopting the ERS method. In [21] a half-mode substrate integrated waveguide was presented with radiation null using ERS method. However, both of the designs in [20] and [21] were based on uniform LWAs with continuous radiation apertures. In [22], a preliminary study on the constituents of the near field of a periodic-slotted leaky waveguide is presented. In this paper, we report a systematic method for generating radiation null in the radiation pattern of a periodic LWAs from the perspective of ERS. A periodic-strip LWA with high-gain pencil beam is developed, and a radiation null in prescribed direction is realized by controlling the ERSs of the radiating mode. This method has little influence on the gain and beamwidth, and only needs simple structural modifications to achieve pattern synthesis.

This paper is organized as follows. In Section II, using a leaky wave structure of periodic metal strips printed on a grounded substrate, the mode expansion method and the concept of ERSs are described in detail, and the method of generating radiation null for periodic LWA is developed. In Section III, the result of mode expansion is given, and a periodic LWA with radiation null in the pattern is designed and fabricated. The measured results are given to verify the antenna performance. Finally, Section IV concludes the paper.

\section{CONFIGURATION AND THEORY}

The basic configuration of the developed LWA is shown in Fig. 1(a). It is implemented on a two-layer substrate of Rogers RT5880 laminates, with dielectric constant of 2.2 and total thickness of 3.15 $\mathrm{mm}$. The thickness of the copper layer is $18 \mu \mathrm{m}$. The antenna is composed of two parts: the first part is the feeding structure, consisting of a planar parabolic reflector (with profile expression $x^{2}$ $=4 F z$ ), a coaxial feeding probe (with height of $1.9 \mathrm{~mm}$ ) and an inductive reflective post (connecting the top and bottom metal 


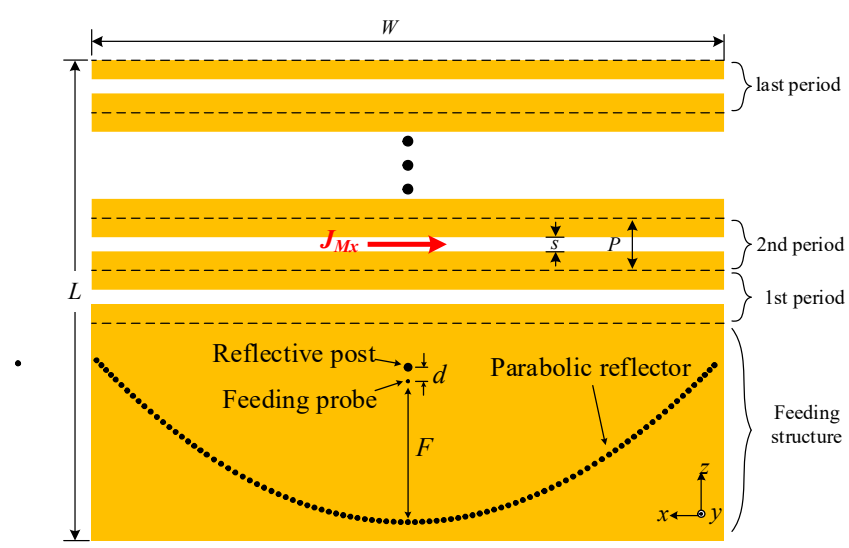

(a)

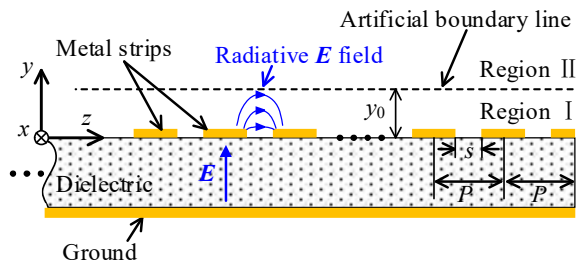

(b)

Fig. 1. Configuration of the one-dimensional periodic structure. (a) Top view. (b) Sectional view of the periodic metal strips. (Not to scale).

plates), which operates based on the parabolic reflector principle similar to that in [23] and our previous work in [24]; the second part is a one-dimensional structure of periodic metallic strips. There are 30 periods in total, and the center of each period is set at the center of the slot. The width of the last strip is only half the width of the other strips to maintain the same period length $P$. The phase distribution along each slot is uniform due to the same phase distribution at the aperture of the parabolic reflector. The ground covers the whole bottom of the substrate. The structural parameters in Fig. 1 are (mm): $d=3.0, F=30.3, P=6.5, s=2.0, W=136$, and $L=252.5$. The E field inside the dielectric is mainly polarized in $y$ direction, whilst at the slot the E field is mainly polarized in $z$ direction, as depicted in Fig. 1(b).

The radiation property of the presented periodic LWA is analyzed using the model shown in Fig. 1(b). The upper half space in yoz plane is divided into two regions (named Region I and Region II) by an artificial boundary line above the structure with height $y_{0}=0.5$ $\mathrm{mm}$. The field $E^{\mathrm{I}}$ in Region I is calculated with the full-wave method. The field $E^{\mathrm{II}}$ in Region II can be expressed as the following equations using the mode expansion method [22]

$$
\left\{\begin{array}{l}
E_{x}^{\mathrm{II}}=\sum_{n=-\infty}^{\infty}\left(B_{n} \omega \mu_{0} \beta_{y n}\right) e^{-j \beta_{z n} z} e^{-j \beta_{y n y} y} \\
E_{y}^{\mathrm{II}}=\sum_{n=-\infty}^{\infty}\left(\mathrm{A}_{n} \beta_{y n} \beta_{z n}\right) e^{-j \beta_{z n} z} e^{-j \beta_{y y} y} \\
E_{z}^{\mathrm{II}}=\sum_{n=-\infty}^{\infty} \mathrm{A}_{n}\left(\mathrm{k}_{0}^{2}-\beta_{z n}^{2}\right) e^{-j \beta_{z z n} z} e^{-j \beta_{y n} y}
\end{array}\right.
$$

where $k_{0}$ is the propagation constant in free space, $n$ represents the $n^{\text {th }}$ spatial harmonic $(\mathrm{SH}), A_{n}$ and $B_{n}$ are unknown coefficients. $\beta_{z n}$ and $\beta_{y n}$ are the propagation constants of the $n^{\text {th }} \mathrm{SH}$ in $z$ and $y$ directions, respectively, and can be expressed as

$$
\left\{\begin{array}{l}
\beta_{z n}=\beta_{z, 0}+2 n \pi / P \\
\beta_{y n}^{2}=k_{0}^{2}-\beta_{z n}^{2}
\end{array}\right.
$$

where $\beta_{z, 0}$ represents the phase constant of the fundamental mode in the periodic structure.

Continuity of the tangential components of the fields must be satisfied on the artificial boundary. Considering the structural characteristics, the component $E_{x}$ on the boundary is so weak that it can be ignored for simplicity, and only the component $E_{z}$ needs to be taken into account, that is

$$
E_{z}^{\mathrm{II}}=\left.E_{z}^{\mathrm{I}}\right|_{y=y_{0}}
$$

Then by picking up sample points at $(2 N+1)$ nodes uniformly distributed in $z$ direction within one period, the unknown coefficients $A_{n}$ and $B_{n}$ can be achieved using the matrix solving method in [22], and hence each SH can be determined using Eq.(1).

Based on the above analysis on mode expansion, we present a new method for generating radiation null region in the radiation pattern of a periodic LWA by controlling the radiation strength of ERSs corresponding to the radiating mode. Firstly, the calculation of radiation for the periodic LWA starts from the equivalent magnetic current $J_{M x, n}$ corresponding to each $\mathrm{SH}$, which can be expressed as

$$
J_{M x, n}(z)=J_{M x, n}(0) \cdot e^{-j \beta_{z, n} z} e^{-\alpha z}
$$

Since only the component $E_{z}$ is considered, the equivalent magnetic current ( $J_{M x}$ in Fig. 1(a)) has only $x$ component, and propagates in $z$ direction. In addition, because the magnitude of each $\mathrm{SH}$ is proportional to the magnitude of the total periodic field at the antenna aperture, all the SHs have the same attenuation constant $\alpha$. It is worth noting that although the leakage rate is varied periodically in $z$ direction due to the structure periodicity, $\alpha$ can still be defined as the average attenuation constant per unit length. So the radiation of the periodic aperture field can be achieved using the integrations of all the magnetic currents as in Eq.(5).

$$
E(\theta)=C \sqrt{\alpha} e^{-j k_{0} r} e^{j k_{0} y_{0} \sin \theta} \cdot \sum_{n=-N}^{N-1} \int_{0}^{L} J_{M x, n}(z) e^{j k_{0} z \cos \theta} d z
$$

where the coefficient $C$ is proportional to $-\sqrt{\frac{j k_{0} P_{0}}{4 \pi r}}, r$ represents the field point in the far field region, and $P_{0}$ is the incident power. Although all the SHs have been considered in Eq.(5), in fact the far field is dominated by the terms of radiating modes, while the other terms of integrations have little contributions due to slow-wave properties.

The ERSs have already been applicable for the analysis of uniform travelling wave structures [20], [21]. However, it cannot be adopted straightforwardly for a periodic structure. The periodic aperture field can be expanded into infinite terms of SHs, and each $\mathrm{SH}$ can be regarded as a travelling wave, as expressed in Eq.(5). Therefore, in this way the ERSs can be applied to each SH. Based on this principle, the total radiation of a periodic aperture field is expressed in Eq.(6) as a summation of the radiations of all the SHs, in which $L_{e 0, n}$ and $L_{e, n}$ are the location and length of the ERS corresponding to the $n^{\text {th }}$

$$
\begin{aligned}
E(\theta)= & C \sqrt{\alpha_{E R S}} e^{-j k_{0} r} e^{j k_{0} y_{0} \sin (\theta)} \cdot \sum_{n=-N}^{N-1}\left(\int_{L_{e 0, n}(\theta)-L_{e, n}(\theta)}^{L_{e 0, n}(\theta)}+\int_{L-L_{e 0, n}(\theta)}^{L-L_{e 0, n}(\theta)+L_{e, n}(\theta)}\right) J_{M x, n}(z) e^{j k_{0} z \cos (\theta)} d z \\
& +C \sqrt{\alpha} e^{-j k_{0} r} e^{j k_{0} y_{0} \sin (\theta)} \cdot \sum_{n=-N}^{N-1}\left(\int_{L_{e 0, n, n}(\theta)-L_{e, n}(\theta)}^{L_{e 0, n}(\theta)}+\int_{L_{e 0, n}(\theta)-L_{e, n}(\theta)}^{L_{e 0, n}(\theta)}+\int_{L-L_{e 0, n}(\theta)}^{L-L_{e 0, n}(\theta)+L_{e, n}(\theta)}\right) J_{M x, n}(z) e^{j k_{0} z \cos (\theta)} d z
\end{aligned}
$$


$\mathrm{SH}$, according to the definition in [19], and $\alpha \mathrm{ERS}$ is the attenuation constant on the ERSs. In fact the first term in Eq.(6) represents the radiation of ERSs, while the second term represents the radiation of other sections. If $\alpha \mathrm{ERS}=\alpha$, the summation of the two terms is exactly the same as Eq.(5). If $\alpha_{\mathrm{ERS}}<\alpha$, it means that a suppression is imposed on the ERSs, generating smaller radiation strength on the ERSs than that on other sections. Particularly, if $\alpha_{\mathrm{ERS}}=0$, the radiation of ERSs will be completely eliminated.

In Eq.(6), it is worth noting that not all the SHs are radiating modes. Suppression on the ERSs of slow-wave modes will have weaker effect on the far field radiation when they have smaller ERSs than that of radiating modes. Under this condition, the manipulation on the radiating modes will have dominant impacts on the radiation pattern. By suppressing the ERSs of the radiating mode in a specific direction, a radiation null region is expected to be generated in this direction. In this paper the $n=-1 \mathrm{SH}$ is set to be the only radiating mode, so the mono-harmonic radiation condition as following is required:

$$
\left\{\begin{array}{l}
\beta_{z,-2}<-k_{0} \\
\beta_{z, 0}>k_{0}
\end{array}\right.
$$

which can be further derived as

$$
P<\frac{4 \pi}{k_{0}+\beta_{z, 0}}
$$

Thus in Eq.(6) only the term of $n=-1$ SH needs to be considered. If the $n=-1$ SH can be controlled, the overall radiation of the antenna can be controlled as well since all the other modes are non-radiative except the $n=-1 \mathrm{SH}$.

It is worth mentioning that, generally speaking, the $n=-1 \mathrm{SH}$ cannot be controlled independently because it is part of the total periodic field, and it cannot exist independently of the other SHs. So the manipulation on the $n=-1 \mathrm{SH}$ needs to be achieved through the control of the total periodic field, which has an effect on all the other SHs.

\section{RESULTS AND DISCUSSION}

In this paper the simulation is conducted using the commercial software CST Microwave Studio. The period length $P$ of the strips is set to be $6.5 \mathrm{~mm}$. A design at $23 \mathrm{GHz}$ is selected as an example to demonstrate the method for generating radiation null. Through the full-wave simulation, the phase constant $\beta_{z, 0}$ and the attenuation constant $\alpha$ of fundamental mode at $23 \mathrm{GHz}$ are obtained, which are $\beta_{z, 0}=665 \mathrm{rad} / \mathrm{m}$ and $\alpha=9.1 \mathrm{~Np} / \mathrm{m}$. Under this condition, it can be inferred that the mono-harmonic radiation condition in Eq.(8) is satisfied.

\section{A Aperture Field Expansion by Mode Method}

Although the mode expansion method in Section II is rigorous only for the case without any attenuation along the structure $(\alpha=0)$, it can still be approximately adopted due to the small attenuation in the real model $\left(\alpha / k_{0}=0.019, k_{0}=482 \mathrm{rad} / \mathrm{m}\right)$. Then using the presented method, the simulated aperture field of the periodic LWA is expanded into SHs. We set $N=25$ to ensure that the sampling density of the aperture field is sufficient for the computational accuracy of the mode expansion method. Then the magnitudes of all the SHs are achieved, as shown in Fig. 2(a). It is worth noting that the height $y_{0}$ of the artificial boundary line is set to be $0.5 \mathrm{~mm}$, which is helpful to obtain more information of higher-order SHs than that by setting a higher $y_{0}$, since higher-order SHs are usually bounded modes. It can be seen that the $n=-1$, and $n=0$ SHs have larger

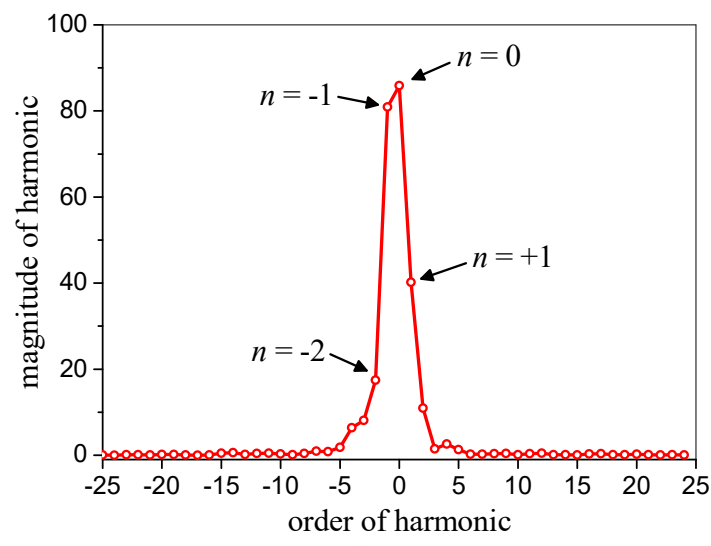

(a)

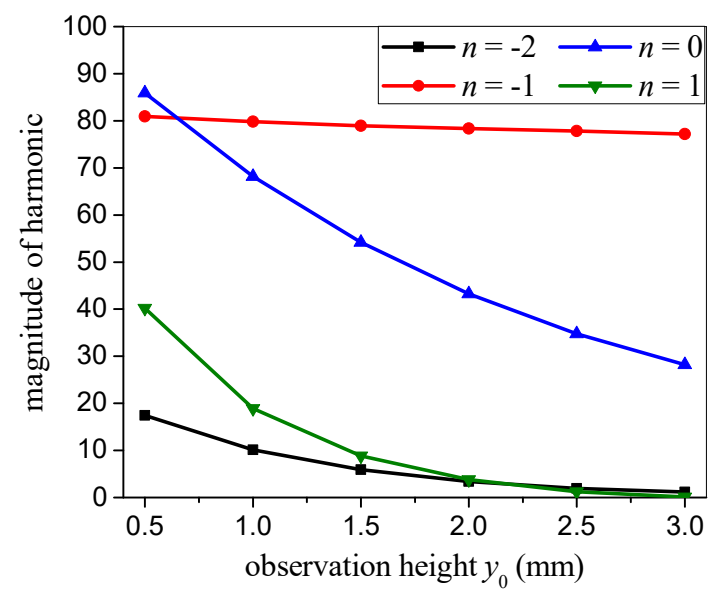

(b)

Fig. 2. Magnitudes of the SHs. (a) Magnitudes of SHs at the height $y_{0}=0.5$ $\mathrm{mm}$. (b) Magnitudes of four SHs as functions of the height $y_{0}$.

magnitudes than the other SHs, demonstrating that they possess more power than the other SHs. However, only the $n=-1 \mathrm{SH}$ is a radiating mode, while all the other SHs are bounded modes. Therefore, it is inferred that most power is being carried by the bounded modes propagating along the antenna surface, while the power carried by the $n=-1 \mathrm{SH}$ is being leaked out to the free space. On the other hand, if the aperture field is observed at a varied height $y_{0}$, the magnitudes of four SHs $(n=-2,-1,0,+1)$ are depicted in the Fig. 2(b). It is illustrated that the near field is contributed mostly by the lower order SHs. When the observation height $y_{0}$ increases, the magnitudes of bounded modes will decrease dramatically, while the magnitude of the radiating mode $(n=-1)$ keeps almost unchanged. Therefore, the far field is dominated by the radiating mode. In addition, it can be inferred that if the ERSs of the radiating mode can be manipulated, the total radiation of the antenna can be controlled as well.

\section{$B$ Generation of the Radiation Null Region}

According to the above analysis, the far-field radiation of the periodic LWA is mainly generated by the ERSs of the $n=-1$ SH. Therefore, by suppressing the ERSs of the $n=-1 \mathrm{SH}$, the far-field radiation around a specific direction can be depressed, and hence a radiation null region is expected to be generated.

The procedure of the method is illustrated as follows: 


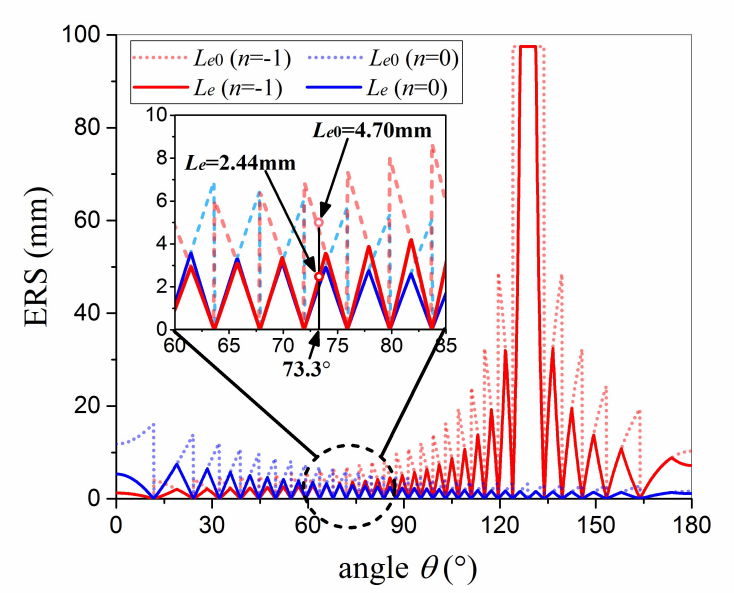

Fig. 3. The ERSs of different SHs as functions of angle $\theta$.

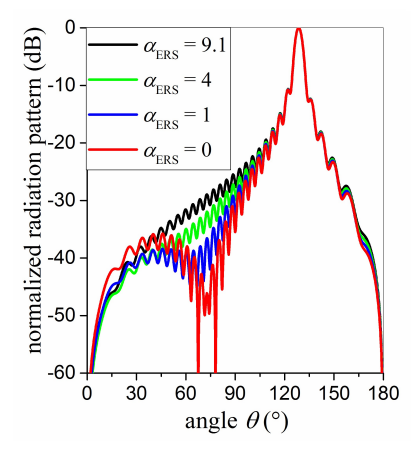

(a)

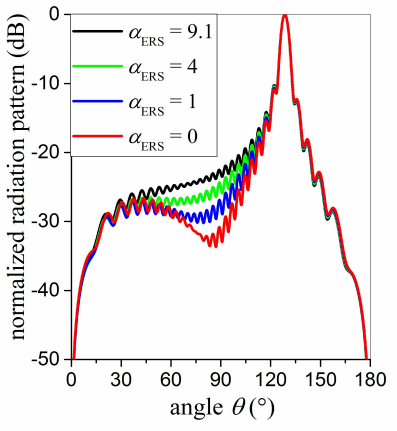

(b)
Fig. 4. The normalized radiation pattern with different $\alpha_{\mathrm{ERS}}$. (a) only considering the $n=-1 \mathrm{SH}$ (b) considering both of the $n=-1$ and $n=0$ SHs.

(1) The phase constant for the basic mode is achieved from fullwave simulation, that is $\beta_{z, 0}=665 \mathrm{rad} / \mathrm{m}$. The phase constant $\beta_{z, n}$ of each SH is calculated by substituting $\beta_{z, 0}$ into Eq.(2).

(2) The ERSs for the SHs are calculated using Eq.(4) in [19], and the results as a function of the observation angle $\theta$ are achieved, as shown in Fig. 3. The ERSs of the $n=-1$ and $n=0$ SHs are shown, and we can know all the information including the length $\left(L_{e}\right)$ and location $\left(L_{e 0}\right)$ of ERSs for these SHs. It can be seen that the ERS for the $n=-1 \mathrm{SH}$ is much larger than that for the $n=0 \mathrm{SH}$, especially around the main beam direction, since the main beam is generated by the $n=-1 \mathrm{SH}$ (the main beam direction is $\theta=128.6^{\circ}$, which is consistent with the result using $\left.\theta=\arccos \left(\beta_{z,-1} / k_{0}\right)\right)$. However, the ERSs of the $n=0$ and $n=-1$ SHs are comparable in the angular range from $60^{\circ}$ to $80^{\circ}$. Therefore, it can be indicated that when the observation angle $\theta$ is smaller than $60^{\circ}$, the $n=0 \mathrm{SH}$ with larger ERSs has greater contribution to the radiation than that of the $n=-1$ $\mathrm{SH}$. On the other hand, the radiation will be mainly contributed by the $n=-1$ SH when $\theta$ is larger than $80^{\circ}$.

(3) Assume that the desired radiation null region is around the direction $\theta=73.3^{\circ}$. Then according to Fig. 3 we can know the location and length of the ERSs for the $n=-1 \mathrm{SH}$, that is $L_{e}=4.70$ $\mathrm{mm}$ and $L_{e}=2.44 \mathrm{~mm}$ corresponding to the direction $\theta=73.3^{\circ}$. By reducing the leakage constant $\alpha_{\text {ERS }}$ of the ERSs, we can introduce a null region into the radiation pattern. Fig. 4 (a) shows the results obtained by considering only the term of $n=-1$ in Eq.(6), whilst in Fig. 4 (b) both of the $n=-1$ and $n=0$ SHs are considered. It can be seen that when $\alpha$ ERs is reduced, an obvious null region occurs in both (a) and (b). The null depth gets deeper as $\alpha$ ERS decreases, and the maximum depth can be achieved when $\alpha_{\mathrm{ERS}}$ is zero, since the radiation of the ERSs of the $n=-1 \mathrm{SH}$ corresponding to this angle is completely suppressed. However, due to the influence of the radiation from $n=0 \mathrm{SH}$, the radiation null in Fig. 4 (b) is not so good as that in Fig. 4 (a), especially in small angle $\theta<70^{\circ}$, since in this range the contribution of the $n=0 \mathrm{SH}$ cannot be ignored, as already claimed in procedure (2).

Note that all the above analysis is based on the theoretical models of ideal magnetic currents, and the results are achieved using the theoretical formulae. In the following section, a practical periodic LWA will be designed using the proposed method.

\section{Application to a Practical Periodic LWA}

Based on the above theoretical results, in this section the method for generating a radiation null in the radiation pattern is applied to a practical periodic LWA. As mentioned earlier, the $n=-1 \mathrm{SH}$ is just one component of the periodic aperture field, and cannot be manipulated independently of the aperture field. So the manipulation is imposed on the whole aperture field by changing the structure, and in this way the $n=-1$ SH can be changed along with the total aperture field. Thus, first we need to find the location of ERSs in the antenna structure (in Section III-B we know that $L_{e 0}=4.70 \mathrm{~mm}$ and $L_{e}=2.44 \mathrm{~mm}$ ). Fig. 5 shows the relationship between the ERSs and the periodic structure. It can be seen that the ERSs locate closely to the two ends of the structure but not at the ends. The first and last slots are included in the ERSs regions. Therefore, it can be inferred that if the widths of the first and the last slots (represented with $s^{\prime}$ in Fig. 5) are simultaneously reduced, the aperture fields at these two slots can be suppressed to some extent, resulting in a reduced magnitude of the $n=-1 \mathrm{SH}$ at these locations.

Although the location and width of the slot do not exactly match with the ERS locations, it still has effect on the generation of radiation null. Fig. 6 shows that the radiation patterns of gain with varied width $s^{\prime}$ for the first and last slots, obtained by the full-wave simulation using CST. It can be observed that when $s^{\prime}$ decreases from $2.0 \mathrm{~mm}$ to $0.4 \mathrm{~mm}$, the radiation strength within the angular range around $\theta=73.3^{\circ}$ is depressed, and a radiation null region occurs. A maximum reduction of $19 \mathrm{~dB}$ is obtained in the radiation null region when $s^{\prime}=0.4 \mathrm{~mm}$. Meanwhile, the method has little influence on the gain and beamwidth of main beam. The gain is 29.3 $\mathrm{dB}$ when $s^{\prime}=0.4 \mathrm{~mm}$. However, since deeper radiation null requires narrower slot width $s^{\prime}$, there might be a trade-off between the specification and implementation based on abrupt variation of structure and practical processing precision, which is similar to the case in [2].

It is worth noting that in theory the $\mathrm{SH}$ is derived from a periodic field that is infinitely long with identical magnitudes in all the periods, and with the same phase difference between any adjacent periods. Therefore, an ideal SH is a uniform plane wave with infinitely long aperture, and without any attenuation or suppression along the aperture. However, the method in this paper is proposed for antennas with finite apertures, and is developed based on the assumption that the strength of $\mathrm{SH}$ can be manipulated in some specific locations. That is because the ERS method can be used for any travelling-wave structure with finite length, and the SH modes can be approximately regarded as travelling waves. Although this is different from rigorous mathematic solutions, it shows good result of radiation null in the radiation pattern of a practical design of periodic LWA.

According to Fig. 6, the LWA with $s^{\prime}=0.4 \mathrm{~mm}$ for the widths of the first and last slots is selected as the final design. It is fabricated 


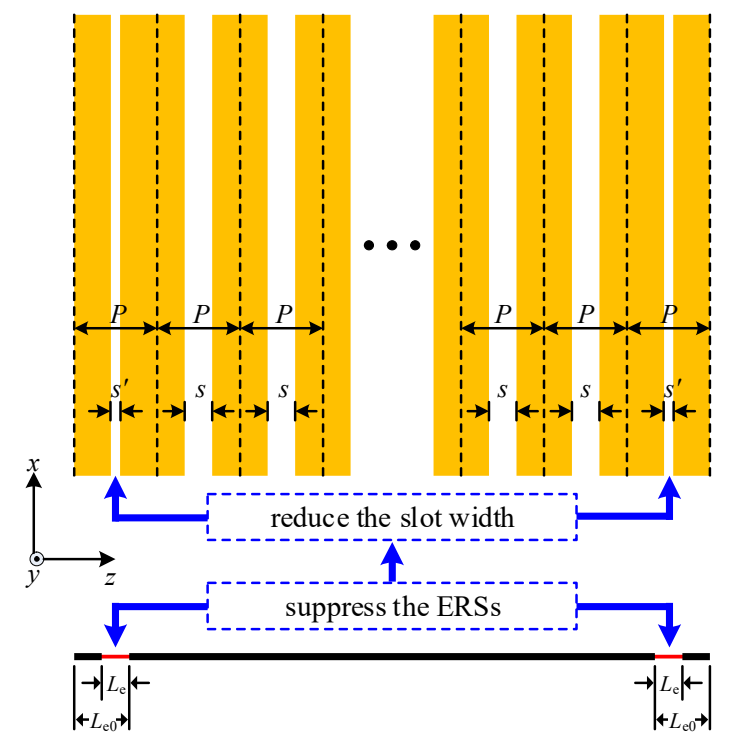

Fig. 5. The location of ERSs in the periodic LWA.

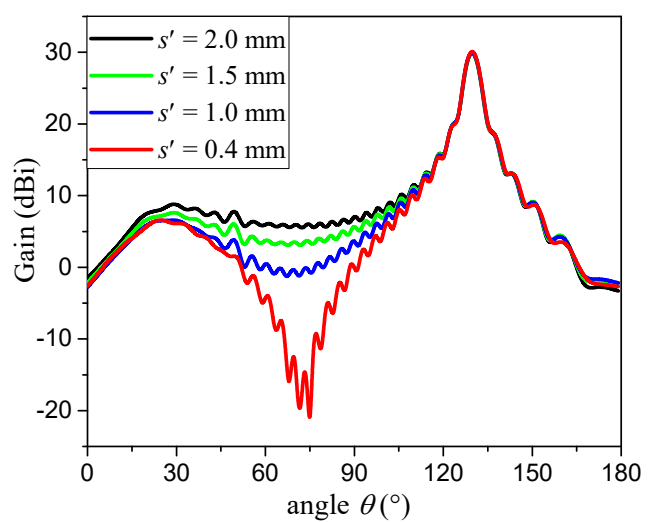

Fig. 6. The full-wave simulated radiation patterns of gain with different width $s^{\prime}$ of the first and last slots.

through PCB processing, and another antenna (all the slots have the same width) are also fabricated as an original design for comparison. The photographs of the fabricated antennas are shown in Fig. 7. The reflection coefficients of both antennas are depicted in Fig. 8. It can be found that both the original antenna and the new design with radiation null have similar frequency bands from $21.5 \mathrm{GHz}$ to 30 GHz. The measured results are also given, showing a slight shift to higher frequency. It may be caused by the slight fabrication error, and particularly there may be some effects of parasitic parameters at the probe feeding point inside the parabolic structure, which will lead to frequency shift.

The measured radiation patterns of gain in E plane (yoz plane) at $23 \mathrm{GHz}$ are shown in Fig. 9. A radiation null region can be clearly observed within the angular range around $\theta=73.3^{\circ}$ in the pattern of the presented LWA, which demonstrates the validation of the proposed method. The measured gain for the original LWA is 28.5 $\mathrm{dBi}$ with a $3 \mathrm{~dB}$ beamwidth of $5.0^{\circ}$, while the measured gain is 28.1 $\mathrm{dBi}$ for the proposed LWA with radiation null, with a $3 \mathrm{~dB}$ beamwidth of $5.3^{\circ}$. Therefore, the gain and beamwidth are not significantly influenced by the proposed method. Fig. 10 depicts the measured radiation patterns in $\mathrm{H}$ plane, which is an inclined plane including the maximum radiation direction. It can be seen that the pattern in $\mathrm{H}$ plane has little change after the radiation null region is produced. However, a slight increase of SLL can be observed, which

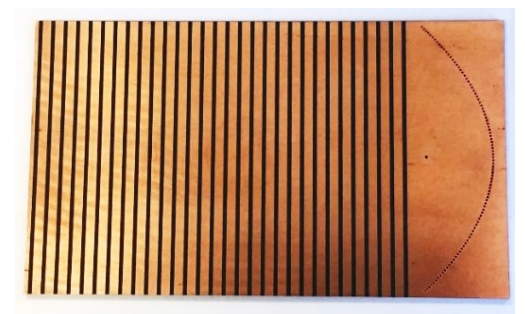

(a)

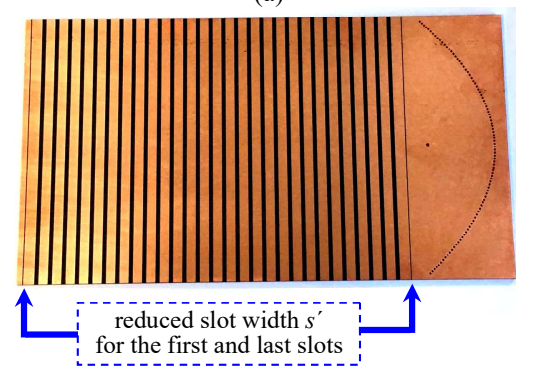

(b)

Fig. 7. Photographs of the fabricated LWAs. (a) original LWA. (b) proposed LWA with narrower slot width $s^{\prime}$ for the first and last slot.

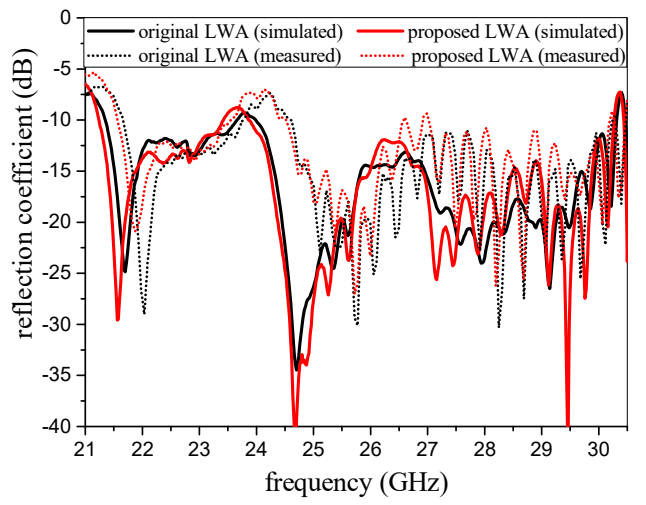

Fig. 8. Simulated and measured reflection coefficients of the original LWA and the proposed LWA with radiation null.

might be caused by the abrupt variation of structure. The $3 \mathrm{~dB}$ beamwidth is increased from $5.0^{\circ}$ (original LWA) to $5.2^{\circ}$ (the LWA with radiation null) in $\mathrm{H}$ plane. In addition, it can be indicated that the presented antenna has a high gain pencil beam. The antenna efficiency is $90.2 \%$ for the original LWA, and $89.0 \%$ for the LWA with radiation null. Therefore, in a summary, the validation of the developed method for generating radiation null for a periodic LWA has been verified by the simulated and measured results. The developed method has the advantages of little influences on the gain, beamwidth and antenna efficiency, and only simple modification on the structure is needed to achieve the radiation null. It is worth noting that the method is developed for synthesis of radiation null in E plane of the antenna, but not in the $\mathrm{H}$ plane. It is more interesting to create radiation null in E plane of the LWA where the higherorder radiation lobe level is usually higher than that in $\mathrm{H}$ plane. In addition, the developed method is executed in a "tapering" manner to periodic unit cells. This "tapering" is independent on the nature of the LWA, which can be uniform, quasi-uniform or periodic.

\section{CONCLUSION}

In this paper, a systematic method of generating radiation null for periodic LWAs is developed from a new perspective, involving the 


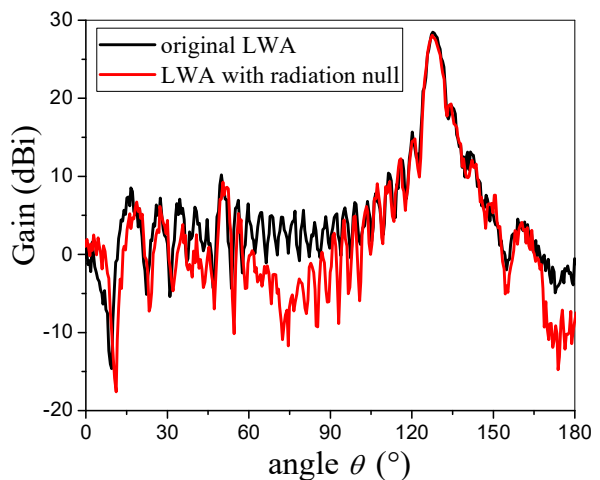

Fig. 9. The measured radiation patterns of gain in E plane at $23 \mathrm{GHz}$ for the original LWA and the proposed LWA with radiation null.

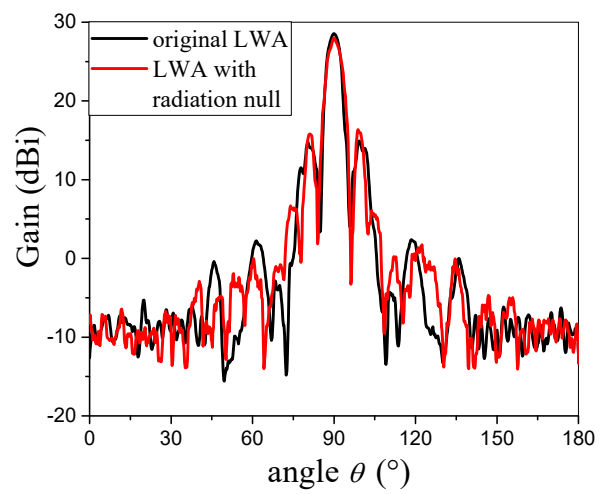

Fig. 10. The measured radiation patterns of gain in $\mathrm{H}$ plane at $23 \mathrm{GHz}$ for the original LWA and the new design with radiation null.

mode expansion method and the ERS method. By using the proposed method, a periodic LWA fed with a parabolic structure is presented, with a radiation null in the radiation pattern. The antenna is fabricated and measured. Both the simulated and experimental results show an obvious radiation null within the angular range around $\theta=73.3^{\circ}$. The measured gain is $28.1 \mathrm{dBi}$, and it is illustrated that the proposed method does not have much impact to the gain, the beamwidth and the antenna efficiency.

It should be noted that most of the existing methods of generating radiation null for LWAs are for uniform structures, so the method for periodic LWAs presented in this paper would serve to fill a gap in this research area. A salient advantage of the presented method is that it is easy to be implemented, as it only needs simple structural modifications to achieve good result of radiation null.

\section{REFERENCES}

[1] A. A. Oliner and D. R. Jackson, "Leaky-Wave Antennas," in J. L. Volakis (ed.), Antenna Engineering Handbook, Fourth Edition, New York, McGraw-Hill, 2007, Chapter 11.

[2] J. L. Gomez-Tornero, A. J. Martinez-Ros, R. Verdu-Monedero, "FFT synthesis of radiation patterns with wide nulls using tapered leaky-wave antennas," IEEE Antennas Wireless Propa. Lett., vol. 9, pp. 518-521, May 2010.

[3] A.Martinez-Ros, J. Gómez-Tornero, and G. Goussetis, "Holographic pattern synthesis with modulated substrate integrated waveguide linesource leaky-wave antennas," IEEE Trans. Antennas Propag., vol. 61 no. 7, pp. 3466-3474, Jul. 2013.

[4] B. B. Tierney, and A. Grbic, "Arbitrary beam shaping using 1-D impedance surfaces supporting leaky waves," IEEE Trans. Antennas Propag., vol. 63, no. 6, pp. 2439-2448, Jun. 2015.

[5] N. Nguyen-Trong, L. Hall, and C. Fumeaux, "Transmission-line model of non-uniform leaky-wave antennas," IEEE Trans. Antennas Propag., vol. PP, no. 99, pp. 1-1, 2016.
[6] N. Nguyen-Trong, L. Hall, and C. Fumeaux, "Pattern synthesis with angular mask for leaky-wave antennas," 2016 17th International Symposium on Antenna Technology and Applied Electromagnetics (ANTEM), pp. 1-2, 2016.

[7] Y. J. Cheng, W. Hong, K. Wu, and Y. Fan, "Millimeter-wave substrate integrated waveguide long slot leaky-wave antennas and twodimensional multibeam applications," IEEE Trans. Antennas Propag., vol. 59, no. 1, pp. 40-47, Jan. 2011.

[8] F. L. Whetten and C. A. Balanis, "Meandering long slot leaky-wave waveguide-antennas," IEEE Trans. Antennas Propag., vol. 39, no. 11 , pp. 1553-1560, Jul. 1991.

[9] J. L. Gómez-Tornero, D. Canete-Rebenaque, and A. Álvarez-Melcón, "Printed-circuit leaky-wave antenna with pointing and illumination flexibility," in IEEE Microw. Wirel. Compon. Lett., vol. 15, no. 8, pp. 536-538, Aug. 2005.

[10] J. L. Gomez-Tornero, F. D. Quesada-Pereira, and A. Alvarez-Melcon, "Analysis and design of periodic leaky-wave antennas for the millimeter waveband in hybrid waveguide-planar technology, " in IEEE Trans. Antennas Propag., vol. 53, no. 9, pp. 2834-2842, Sep. 2005.

[11] J. L. Gómez-Tornero, J. Pascual García, and A. Álvarez-Melcón "Efficient full-wave analysis method of leaky-wave modes in periodically loaded dielectric waveguides with application to backwardto-forward frequency-scannable antennas and metamaterials," Int. J. Numer. Model.-Electron. Netw. Device Fields, vol.19, no.2, 173-193, Mar. 2006.

[12] C. Caloz, T. Itoh, "Array factor approach of leaky-wave antennas and application to 1-D/2-D composite right/left-handed (CRLH) structures", IEEE Microw. Wireless Compon. Lett., vol. 14, no. 16, pp. 274-276, Jun. 2004.

[13] J. L. Gómez-Tornero, D. C. Rebenaque, F. Quesada-Pereira, J. P. Martinez and A. Álvarez-Melcón, "PAMELA: a useful tool for the study of leaky-wave modes in strip-loaded open dielectric waveguides," in IEEE Antennas Propag. Mag., vol. 48, no. 4, pp. 54-72, 4 Aug. 2006.

[14] O. Rance, P. Lemaître-Auger, R. Siragusa and E. Perret, "Generalized Array Factor Approach to the Assessment of Discrete Tapered Nonuniform Leaky-Wave Antenna," in IEEE Trans. Antennas Propag., vol. 63, no. 9, pp. 3868-3877, Sep. 2015.

[15] Y. H. Liu, X. Huang, K. D. Xu, Z. Y. Song, S. W. Yang, Q. H. Liu, "Pattern synthesis of unequally spaced linear arrays including mutual coupling using iterative FFT via virtual active element pattern expansion," IEEE Trans. Antennas Propag., vol. 65, no. 8, pp. 39503958, 2017.

[16] X. Y. Ma, Y. H. Liu, K. D. Xu, C. H. Zhu, Q. H. Liu, "Synthesizing multiple-pattern sparse linear array with accurate sidelobe control by the extended reweighted L1-norm minimization," IET Electron. Lett, vol. 54, no. 9, pp. 548-550, May 2018.

[17] R. L. Haupt, "Phase-only adaptive nulling with a genetic algorithm," IEEE Trans. Antennas Propag., vol. 45, no.6, pp. 1009-1015, Jun. 1997.

[18] Y. J. Lee, J. W. Seo, J. K. Ha, et al, "Null steering of linear phased array antenna using genetic algorithm," Microwave Conference, APMC 2009. Asia Pacific, pp. 2726-2729, 2009.

[19] Z. Li, J. H. Wang, Z. Zhang, and M. E. Chen, "Far field computation of the traveling wave structures and a new approach for suppressing the sidelobe levels," IEEE Trans. Antennas Propag., vol. 61, no. 6, pp. 2308-2312, Apr. 2013.

[20] Z. Li, J. H. Wang, J. J. Duan, Z. Zhang, and M. E. Chen, "New approach of radiation pattern control for leaky-wave antennas based on the effective radiation sections," IEEE Trans. Antennas Propag., vol. 63, no. 7, pp. 2867-2878, Jun. 2015.

[21] X. Y. Chen, Z. Li, H. Song, and J. H. Wang, "Generation of radiation null for the HMSIW leaky-wave antenna", IEEE Antennas Wireless Propag. Lett., vol. 16, pp. 2688-2691, 2017.

[22] Z. Li, J. H. Wang, J. J. Duan, Z. Zhang, and M. E. Chen, "Analysis on the radiation property of the bounded modes of periodic leaky-wave structure with finite length using a hybrid method," Scientific Reports 6:22917, 2016.

[23] Z. Chen and Z. Shen, "Wideband flush-mounted surface wave antenna of very low profile," IEEE Trans. Antennas Propag., vol. 63, no. 7, pp. 2430-2438, Jun. 2015.

[24] S. Q. Zhang, Z. Li, J. H. Wang, "A novel SIW H-plane horn antenna based on parabolic reflector," Int. J. Antennas Propag., vol. 2016, Article ID 3659230, 2016. 